E.G. Kilinc, B. Canovas, F. Maloberti, and C. Dehollain, "Intelligent Cage for Remotely Powered Freely Moving Animal Telemetry Systems", IEEE Proceeding of the International Symposium on Circuits and Systems, pp. 22072210, May 2012.

(C)20xx IEEE. Personal use of this material is permitted. However, permission to reprint/republish this material for advertising or promotional purposes or for creating new collective works for resale or redistribution to servers or lists, or to reuse any copyrighted component of this work in other works must be obtained from the IEEE. 


\title{
Intelligent Cage for Remotely Powered Freely Moving Animal Telemetry Systems
}

\author{
Enver G. Kilinc ${ }^{\star}$, Bastien Canovas ${ }^{\star}$, Franco Maloberti ${ }^{\diamond}$, and Catherine Dehollain ${ }^{\star}$ \\ ${ }^{\star}$ RF-IC Group, Ecole Polytechnique Fédérale de Lausanne, Lausanne, Switzerland \\ $\diamond$ Integrated Microsystem Laboratory, Universitá degli Studi di Pavia, Pavia, Italy \\ Email: enver.kilinc@epfl.ch, franco.maloberti@unipv.it, catherine.dehollain@epfl.ch
}

\begin{abstract}
An intelligent power feedback design for remotely powered freely moving animal telemetry systems is described. The system uses an optimized wireless power transmission that utilizes multiple transceiver coils. An intelligent mouse cage with controller unit of the feedback system are proposed. System description, experimental setup and measurements of the remotely powered implantable monitoring system are presented.
\end{abstract}

\section{INTRODUCTION}

Nowadays, the advances in microelectronics make remotely powered telemetry systems widespread. These systems are designed to be implanted mainly for animals. Especially, implantable telemetry system for the laboratory mouse is quite essential because the laboratory mouse is used for developing new treatments for the diseases and research activities since their DNA sequence is close to human [1]. However, designing a remotely powered implantable telemetry system for laboratory mouse has certain challenges. The major challenge is that, the system implanted inside the mouse must be extremely small and light-weight. In addition, the system must be fast enough to detect rapid mouse movement and respond to it quickly.

The remotely powered monitoring system is comprised of two major modules. First module is the external reader which sends the commands to the internal unit and receives data from the internal unit. Second module is the internal side which is the implanted part acquiring data from the sensors. In order to activate the implanted part, the wireless power transmission should be sustained continuously. A battery can be used to secure a continuous operation when the power transmission is insufficient. However, the batteries require higher volume compared to the circuits and also they increase the overall weight. In addition, the batteries should be replaced at the end of the their lifetime which requires additional medical surgeries. Therefore, it is desirable to use batteryless implanted systems with continuous wireless power transmission.

\section{Wireless Power TRANSMission}

The most common methods for wireless power transmission use electromagnetic waves or magnetic field. The use of electromagnetic waves enables power harvesting from relatively long distance $(1-10 \mathrm{~m})$. However, using magnetic field is more effective when the distance between external and implanted units is short (a couple of decimeters) [2]. Therefore, power can be transferred more efficiently by using magnetic field at

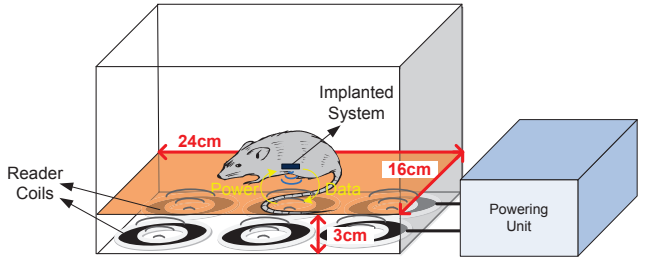

Fig. 1. Remotely powered implantable telemetry system for laboratory mouse

short distances. Fig. 1 shows a remotely powered implantable sensor system for a laboratory mouse. The power is transferred to implanted unit by employing magnetically coupled inductive link.

In magnetically coupled power transfer systems, most of the power is dissipated during wireless power transmission because the coupling between the coils decreases proportionally with the distance $(d)$ between the coils. Also, the magnetic field strength attenuates proportional to $d^{3}$. Therefore, inductive link efficiency designates the overall efficiency of the system. Consequently, the inductive link has to be designed carefully. The geometries of the reader and implanted coils should be optimized to achieve maximum efficiency by using an optimization tool [3].

The key of the external part is the coil system used to generate the magnetic field carrying power and data. A first solution is to use a single coil under the floor of the cage. For practical cases the size of the cage is large with respect to the mouse as shown in Fig. 1. As a result, placing a single coil under the cage which covers the whole floor decreases the operation frequency of the inductive link due to low self-resonance frequency of the reader coil. The operation frequency has a significant effect on the bandwidth, and hence, the maximum data rate [4]. Also, the induced voltage on the implanted coil is proportional to the operation frequency. Moreover, the maximum magnetic field is generated in the center of the coil. Therefore, the received power decreases drastically when the implanted coil moves from the center to the edges of the reader coil. As a consequence, the implanted unit can shut down due to insufficient power transmission. In order to guarantee the operation of the implanted unit, minimum power required has to be supplied in any situation. Accordingly, the reader coil must work at its maximum level to guarantee the operation therefore, it consumes more power. 


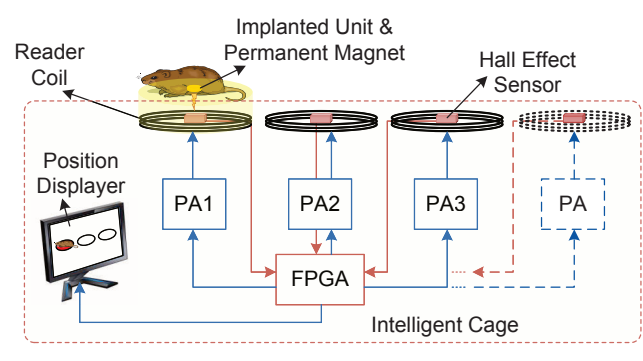

Fig. 2. Intelligent mouse cage system

A more efficient solution is using multiple coils which realize an array of the coils with a relatively high selfresonance frequency. Also, it has been verified in [3], [5] that the optimum reader coil radius is almost $\sqrt{2}$ times the distance between reader coil and implanted coil (supposing the implanted coil is much smaller than the reader coil). Accordingly, an array of optimum reader coils is placed under the cage. Replacing a large coil with smaller coils enables a higher operation frequency and bandwidth and generates higher magnetic field strength. The magnetic field is concentrated at the center of the coils. Increasing the number of the coils will provide a more uniform spatial distribution which prevents positiondependent power transfer efficiency. However, the system may consume more power since the number of coils increases. To avoid this drawback, an intelligent feedback mechanism is proposed to control the power level of the reader coils.

Fig. 2 shows an array of coils which is controlled by the feedback system. By using the feedback mechanism which selects the most appropriate coil among the coils according to the position of the mouse decreases the overall power consumption of the system because only one of the coil in the array will be activated and the rest of the coils will not be energized [6].

\section{Intelligent CAGE}

In telemetry systems aiming at animals, the subject should be able to move freely. Since the limitation of the mobility may induce stress on the subject, the measurement may not be accurate. The subject can be anywhere in the cage during the experiment. Therefore, the mouse should be tracked and the sufficient power should be transferred. Fig. 2 shows the proposed feedback system for remotely powered freely moving mouse telemetry systems.

A small permanent magnet is placed in the center of the implanted coil and linear hall effect sensors are placed in the center of the reader coils. The position of the mouse is detected by using these sensors. The output voltage of the sensors changes according to magnetic field generated by the permanent magnet. For instance, it is assumed that the mouse in Fig. 3 moves from point $A$ to point $C$. At first, the output voltage of $S 1$ sensor is maximized compared to other sensors. However, the output of $S 1$ sensor decreases and the outputs of $S 2, S 4$, and $S 5$ sensors increase when the mouse starts to move from $A$ to $B$. Consequently, the supply

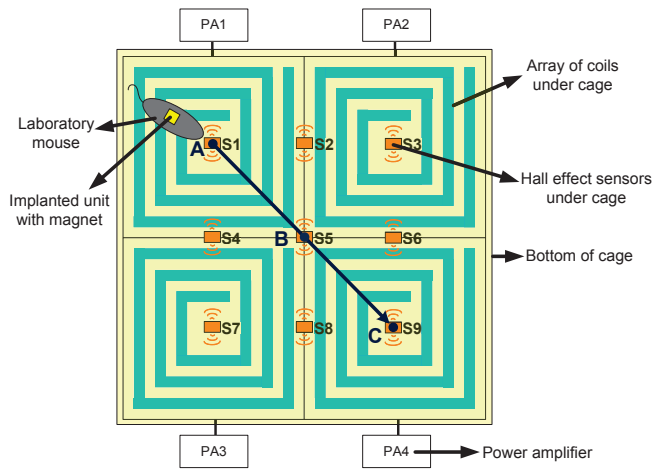

Fig. 3. Feedback system for remotely powered implantable telemetry system

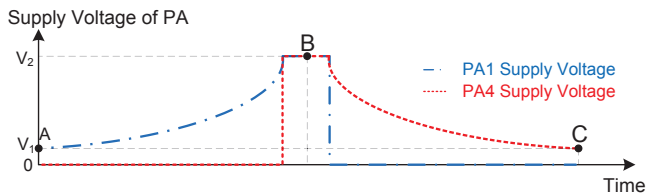

Fig. 4. Supply voltages of power amplifiers with respect to mouse position

voltage of $P A 1$ will be increased. At point $B$, output of $S 5$ is maximized. Therefore, the supply voltage of $P A 1$ and $P A 4$ are also maximized in order to transmit sufficient power. When the mouse passes point $B, P A 1$ will turn off and $P A 4$ will continue to transmit power. Fig. 4 shows the supply voltages of $P A 1$ and $P A 4$ with respect to mouse movement from point $A$ to $C$. By looking at the output of the different hall effect sensors, the coordinates of the implanted unit can be identified, and hence, appropriate coil in the array will be turned on and sufficient power will be transferred.

In order to supply sufficient power, the position of the implanted coil should also be defined according to the center of the reader coil because the power transmission efficiency of the inductive link strongly depends on the magnetic field strength $(H)$ which varies with lateral and also angular misalignments [7]. However, many studies show that the mice spend most of the time in a horizontal position (on their four feet) [1]. Therefore, the demanding misalignment source is lateral which is examined throughout this study. The power transmission efficiency of the inductive link (Fig. 7) with respect to the lateral misalignment which is extracted by using 3D electromagnetic simulator (HFSS) [8] presented in Fig. 5. The transmitted power by the reader unit has to be increased when the internal unit moves from the center to the edges in order to activate the implanted unit. The output of hall effect sensors is also used for defining the position of the implanted coil according to center of the suitable reader coil. Therefore, the adequate power required by the implanted system is transmitted by modifying the supply voltage of the corresponding power amplifier.

\section{Controller Unit}

Fig. 6 shows the block diagram of the controller unit which is developed on an FPGA board. The output voltage of each 


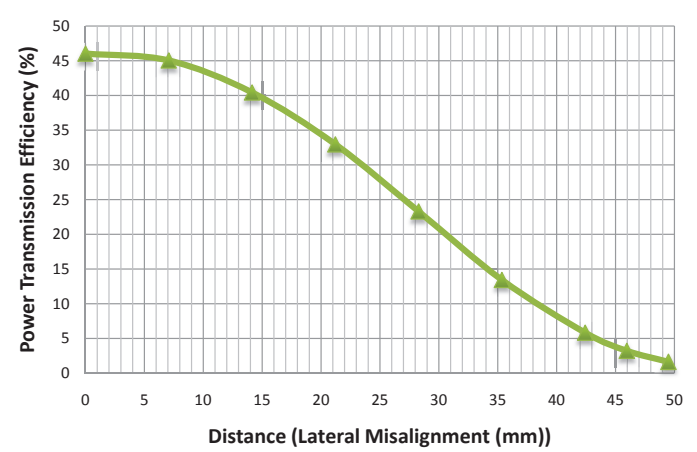

Fig. 5. Power transmission efficiency with respect to lateral misalignment

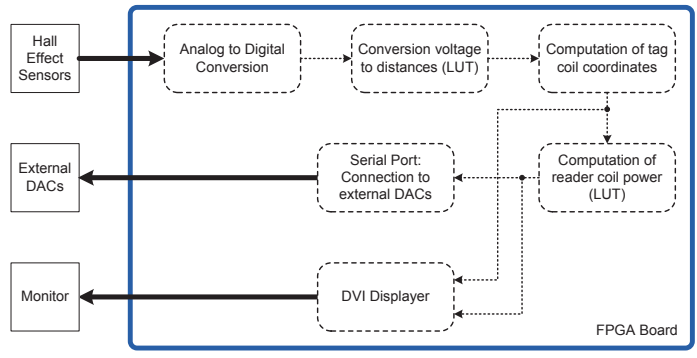

Fig. 6. Block diagram of controller unit

hall effect sensor represents a distance between the magnet and the corresponding sensor. The controller unit reads the output voltages of the hall effect sensors and converts these analog signals to digital signals. Each voltage level has a corresponding distance which is stored in a LookUp Table (LUT). Afterwards, the coordinates of the implanted coil is computed according to the distances obtained previously. In addition, the appropriate reader coil is activated by checking the position of the implanted coil. Furthermore, the position of the implanted coil according to the center of the selected reader coil is also defined in order to transmit sufficient power to implanted unit. The required power is computed by using power transmission efficiency graph which is given in Fig. 5. The calculation of efficiency by a microcontroller is quite difficult since many complex mathematical equations should be calculated such as integrals. Each power level has a corresponding power amplifier supply voltage which is stored in the LUT in the FPGA. Finally, to transmit the required power, the supply voltage of the desired power amplifier is sent digitally to the external DAC.

Aforementioned computations are also used in order to get information about the position of the subject and condition of the coils. Therefore, the movement of the subject is also tracked and displayed by using the same FPGA board.

\section{Intelligent Mouse Cage for Remotely Powered IMPLANTABLE MONITORING SYSTEM}

As a design example, remotely powered implantable monitoring system for a laboratory mouse is chosen. The width and length of the mouse cage are $16 \mathrm{~cm}$ and $24 \mathrm{~cm}$, respectively. The size and weight of the laboratory mouse are approximately

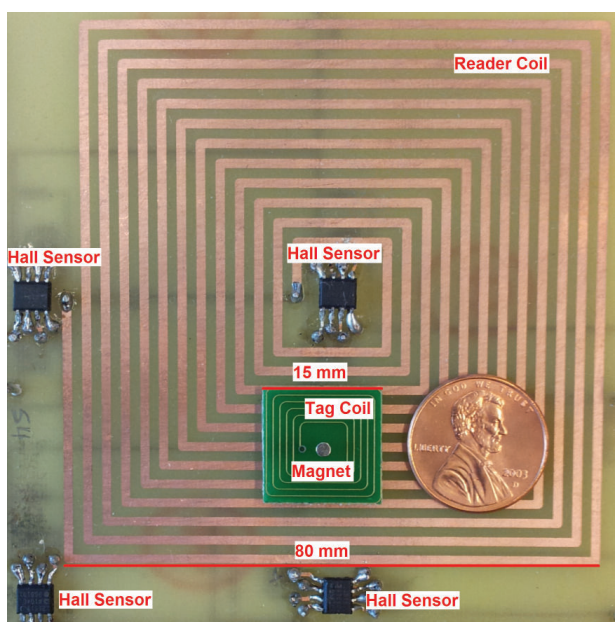

Fig. 7. Optimized inductive link with hall effect sensors

$10 \mathrm{~cm}$ and $40 \mathrm{~g}$. The distance between the reader coil and the implanted unit is $30 \mathrm{~mm}$ vertically. The scenario is represented in Fig. 1.

In order to maximize wireless power transmission, the implanted coil size is chosen as $15 \mathrm{~mm}$ considering the practical aspects of clinical application. The use of the optimization tool [3] gives rise to an efficient inductive link. The optimum reader coil size is $80 \mathrm{~mm}$. Fig. 7 shows the optimized reader and the implanted coils. As the bottom of the cage is rectangular, a square type of coil is preferred to cover all the bottom. Therefore, an array of coils consisting of 6 elements is placed under the cage.

The feedback system uses linear output magnetic field sensors (AD22151, Analog Devices) which have adjustable offset to unipolar or bipolar operation and low offset drift over wide temperature range [9]. The diameter of the chosen permanent magnet is $3 \mathrm{~mm}$ which is placed in the center of the implanted coil as shown in Fig. 7. The magnet is $30 \mathrm{~mm}$ above the sensors' surface. Fig. 8 shows the response of the hall effect sensor for north and south poles of chosen magnet according to lateral misalignment case. The output voltage shows symmetrical behavior for both poles and the control unit is designed for working with both cases. However, the problem is the distance because the output of the hall effect sensor saturates after $30 \mathrm{~mm}$. Therefore, the number of the hall effect sensors is increased in order to cover all the bottom of the cage and detect the position of the mouse correctly. The sensors are placed as in Fig. 7.

The controller unit is implemented on an FPGA with evaluation board (Virtex-5, Xilinx) [10]. The output voltages of the hall effect sensors are converted to 10-bit digital signals by using the ADCs on the evaluation board. In addition, the position displayer is also driven by the DVI output of the same evaluation board. In order to convert the digital signal to analog voltages a 8-channel, 8-bit DAC (DAC8800, Analog Devices) is used externally [11]. The DAC communicates with controller unit by using the serial port of the evaluation board. The DAC requires 11-bit in order to calibrate the required 


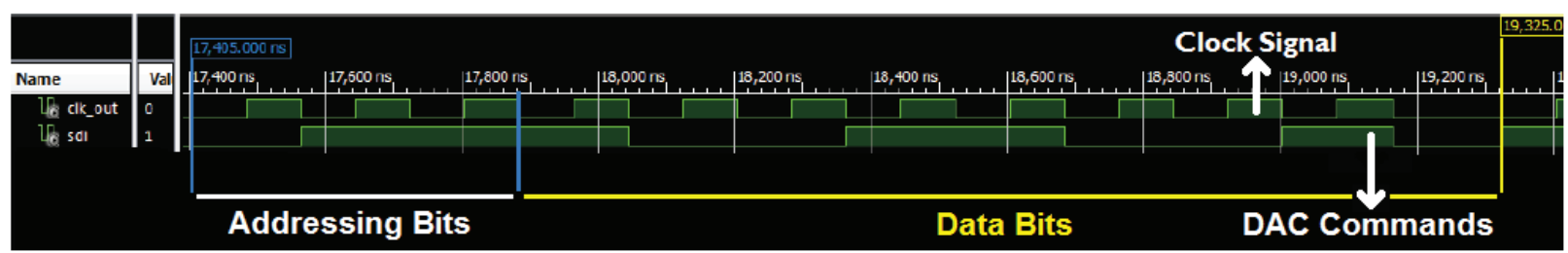

Fig. 9. Signal stream for calibrating DAC

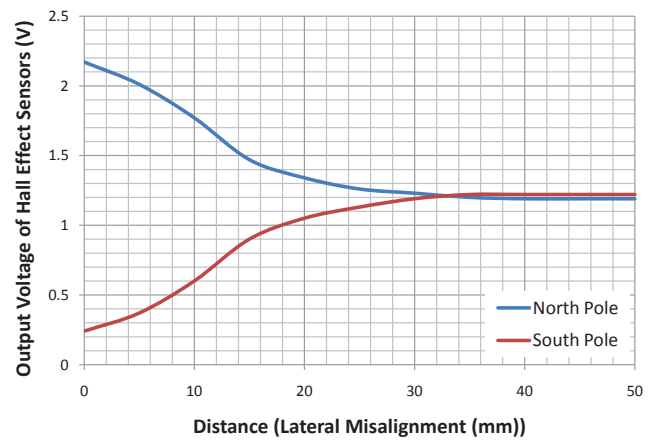

Fig. 8. Hall effect sensor output voltage for north and south poles of magnet

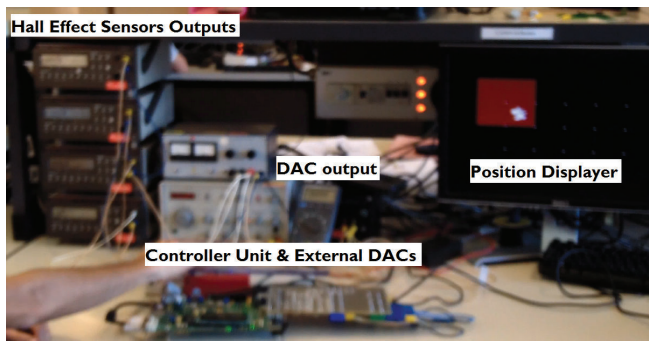

Fig. 10. Experimental test-bench for intelligent mouse cage system

supply voltage of the power amplifier. The first 3 bits stand for selecting the correct channel and the rest 8 bits are used for the voltage value to be converted. Fig. 9 shows the signal stream for calibrating the DAC. The DAC output changes from $0 \mathrm{~V}$ to $3 \mathrm{~V}$ with $11.7 \mathrm{mV}$ step size to control the power amplifier supply. The power amplifier (PA) is chosen a class-E type due to its high efficiency and the operation frequency of the link is $13.56 \mathrm{MHz}$.

The mouse can also be monitored by using the position displayer. In addition, some information about the coils can be obtained by this displayer. Fig. 10 shows the experimental test-bench of the intelligent feedback system and position displayer. In Fig. 10, the red square region represents the activated coil and the white spot demonstrates the position of the mouse on the coil.

In order to cover all the bottom of the cage, the system uses a $2 \times 3$ array of reader coils. 15 hall effect sensors are placed in the center and in the middle of the coils. The total process takes 142 clock cycles with $6.25 \mathrm{MHz}$ clock frequency and the delay is calculated as $22.72 \mu \mathrm{s}$. The previous studies state that refreshment frequency of $10 \mathrm{~Hz}$ is enough for tracking the movement of the mouse [12]. In the proposed feedback system, 12 clock cycles are required for updating one DAC channel and 6 channels are required for remote powering system which has 6 coils. Therefore, the position refreshment frequency is found as $86.8 \mathrm{KHz}$ which is quite sufficient, based on the result in [12].

\section{CONCLUSiON}

This intelligent feedback design is for remotely powered freely moving animal telemetry system. The feedback system tracks the mouse movements by using the hall effect sensors. The control unit which manages the overall system reads the output of the sensors and transmits the sufficient power for the implanted sensor system. Preliminary experimental measurements validate the proposed approach that enables a reduction of the magnetic power received by the mouse. The inefficient power transmission can be a potential risk for a body because the mouse will be exposed to a higher magnetic field which effects body temperature.

\section{ACKNOWLEDGMENT}

This project is supported by Swiss National Funding (SNF) through Sinergia Initiative.

\section{REFERENCES}

[1] J. N. Crawley, What's Wrong With My Mouse: Behavioral Phenotyping of Transgenic and Knockout Mice, 2nd ed. Hoboken,NJ:Wiley, 2007.

[2] D.C. Yates, A.S. Holmes, A.J. Burdett, "Optimal transmission frequency for ultralow-power short-range radio links," in IEEE Trans. Circuits Syst. I, 2004, vol.51, no.7, pp.1405-1413.

[3] E.G. Kilinc, C. Dehollain, F. Maloberti, "Design and optimization of inductive power transmission for implantable sensor system," in Proc. SM2ACD'10, 2010, pp.1-5.

[4] E.G. Kilinc, O. Atasoy, C. Dehollain, F. Maloberti "FoM to compare the effect of ASK based communications on remotely powered systems," in Proc. PRIME'11 Conf., pp.29-32, 2011.

[5] K. Finkenzeller, RFID Handbook, John Wiley \& Sons, 2003.

[6] H.-G. Lim, et. al., "Intelligent pillow type wireless charger for fully implantable middle ear hearing device with a function of electromagnetic emission reduction," in Workshop on Intelligent Information Technology Application, 2008, pp.835-838.

[7] K. Fotopoulou, B.W. Flynn, "Optimum antenna coil structure for inductive powering of passive rfid tags," in IEEE International Conference on RFID, 2007, pp.71-77.

[8] Ansys, Inc., USA. HFSS - 3D Fullwave electromagnetic field simulation. [Online]. Available: http://www.ansoft.com/products/hf/hfss

[9] Analog Devices, Inc., USA. AD22151G - Linear output magnetic field sensor [Online]. Available: http://www.analog.com

[10] Xilinx, Inc., USA. Virtex-5 FPGA family (XC5VFX70T). [Online]. Available: http://www.xilinx.com

[11] Analog Devices, Inc., USA. DAC8800 - Octal 8-bit D/A converter [Online]. Available: http://www.analog.com

[12] N. Chaimanonart, D.J. Young, "A wireless batteryless in vivo EKG and body temperature sensing microsystem with adaptive RF powering for genetically engineered mice monitoring," International Solid-State Sensors, Actuators and Microsystems Conference, 2009, pp.1473-1476. 